\title{
Modelling\& Simulation and Protection\& Control Verification Method for Asynchronous Motor-Synchronous Generator Sets
}

\author{
Shuyang Wang ${ }^{1}$, Qiu Xia ${ }^{1}$, Xingguo Wang ${ }^{1}$, Yarong Guo ${ }^{1}$, Zhengguang Chen ${ }^{1}$, Dingxiang Du ${ }^{1 *}$ \\ ${ }^{1}$ China Electric Power Research Institute, Beijing, 100192, China
}

\begin{abstract}
Power supply system made of asynchronous motor-synchronous generator sets (M-G sets) is often used as power supply for important loads. The system usually consists of two M-G sets. Unlike synchronous generators connecting to large power grid, the two generators in the system can have significant effect on the other when field loss fault occurs. If the protection settings are inappropriate, the two generators may trip one after another during a fault on a single generator, causing the important load losing all the power supply. This paper proposed a simulation and protection verification method based on the RTDS digital modelling for M$\mathrm{G}$ sets including detailed excitation system. The modelling and simulation methods can effectively verify the reasonability of settings of the P-Q restriction of the excitation regulator and the PID controllers, and provide the important basis for the setting of over-current protection and loss of excitation protection, ensuring the safe operation of the power supply system made of M-G sets.
\end{abstract}

\section{Introduction}

A good excitation system can not only ensure high-quality power and the reliability and stability of generator operation, but also effectively improve the technical and economic indicators of generator and its related power system. The serious decline of excitation of large units will lead to the destruction of stability and enter into the state of out of step operation with excitation and generate synchronous power with oscillation. The total field loss generator enters into the state of asynchronous operation, which absorbs a lot of reactive power of the system. There are many researches on field loss and field loss protection for large generator sets connecting to large power grid [18].

The power supply for important loads can be directly supplied by the power grid, but the ability to resist the disturbance of the power grid is poor. The short-term power failure may lead to the power loss of important loads and pose a major threat to the safety of equipment and personnel. For some important equipment in power plants, short-term power loss of some important facilities may lead to a series of unpredictable consequences such as unnecessary unit tripping and grid frequency collapse, so some power plants use asynchronous motor synchronous generator (M-G) sets to supply power to important loads.
The asynchronous motor takes power from the power grid and drives the coaxial flywheel, exciter and synchronous generator rotor to rotate in the same axis. The synchronous generator then supplies power to the load. When the power grid is disturbed for a short time, it will not be conducted to the load. When the power is cut off for a short time, inertia flywheel can maintain the normal power supply to the load for a period of time. When $\mathrm{M}-\mathrm{G}$ set is used for power supply of important load, generally two M-G sets in parallel operation are used as power supply to improve the reliability. Two $M-G$ sets are standby for each other. When one unit is cut off or under maintenance, the other unit can take the maximum load and keep operating. The synchronous generator is generally equipped with differential protection, overcurrent protection, loss of excitation protection, over voltage protection, etc. However, almost none of the generators is equipped with fault recorder, thus making it hard to replay the fault and take proper measures.

Unlike large generator connecting to power grid, for the parallel operation of M-G sets, there are problems such as over-current protection and loss of field protection coordination, $\mathrm{P}-\mathrm{Q}$ restriction and loss of field protection coordination between two $\mathrm{M}-\mathrm{G}$ sets.

According to an accident report from a power plant, the major capacitor in the digital AVR of one of the generators in the M-G sets burned down, causing field loss of the generator. The field loss fault is designated to be cut

*Corresponding author's e-mail: wangshuyang@epri.sgcc.com.cn 
off by the field loss protection, however, the overcurrent protection of the non-faulty generator operated before the filed loss protection, causing two generators trip one after the other, making the important load losing power, causing serious consequences.

So it is necessary to carry out digital modelling of power supply system made of M-G sets, verifying the validity of protection settings and modifying the unreasonable settings according to the simulation results.

A typical power supply system made of two M-G sets is shown as below:

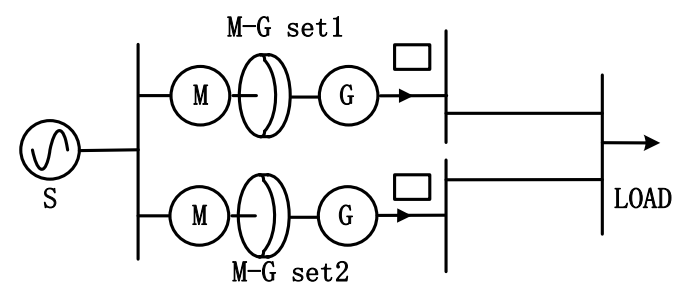

Fig 1. Typical power supply system made of two $M-G$ sets

This paper will introduce the modelling and simulation method designated to verify the settings of various Protection of synchronous machine.

\section{Analysis of protection coordination of M-G sets generators}

\subsection{Coordination of Overcurrent protection\& field loss protection}

Since there are only two synchronous generators operating in parallel, when the excitation system of one generator fails, the other unit will inject large amount of reactive power to the filed lost one thus causing overcurrent. If the setting of protection for two units is inappropriate, the over-current protection action of the non-failing unit may precede the loss of field protection action of the field lost unit, resulting in two generators in the system tripping one after the other, therefore causing the important load losing all power supply, bringing serious consequences to the related equipment, causing economic loss and may even endangering personal safety.

For the M-G sets generator protections discussed in the paper, the over-current protection is usually inverse time current protection, and the field loss protection for small generators is usually low field current protection instead of the R-X field loss protection mostly adopted by large capacity generators. The coordination of the two protection is based on different time delay. To avoid the oscillation, the field loss protection usually has a high time delay while the overcurrent protection operates much faster. The time delay of the field loss protection should not only cooperate with the time delay of the inverse time current protection of the same generator, but also have to cooperate with the time delay of the other generator.

\subsection{Coordination of P-Q limiter\& field loss protection\&}

When the forced excitation of one generator is triggered mistakenly, usually by PT line breaking, the other generator would lower its filed current to receive more reactive power from the forced excitation generator to keep the terminal voltage at the rated level, thus may reach the loss of field operation threshold.

To prevent that situation from happening, the P-Q limiter of the regulator is necessary. P-Q limiter defines the working zone on the P-Q by limiting the field current under different load level. P-Q limiter should ensure that when the generator has to receive reactive power to stabilize the voltage, the field current should not reach the operation threshold of field loss protection.

\subsection{Coordination of over-current\& over-voltage protection}

When the P-Q limiter restrains the further decline of the field current while the force excitation of the other generator is still working, the terminal voltage would inevitably increase, which is harmful for the generators and loads as well. The overcurrent protection should not operate at this point because it may trip both generators.

The operators have to specify the possible overvoltage level to set the appropriate setting value to either trip the over-exciting generator or send warning message to the operators.

\section{Modelling and simulation method}

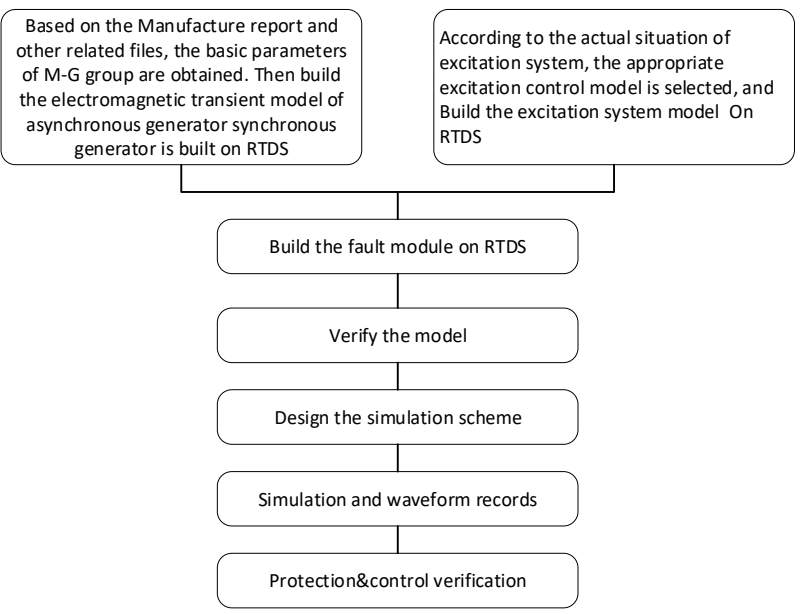

Fig 2. Modelling\& simulation and protection\& control verification flow diagram

Modelling\& simulation and protection\& control verification flow diagram is shown in fig 2 .

To verify the coordination of the protections\& controls mentioned in chapter2, the accurate modelling and comprehensive simulation method is needed. In this paper, a RTDS digital modelling method and simulation scheme for power supply system made of M-G sets are introduced. 


\subsection{RTDS modelling method}

(i) Obtain the factory test report of asynchronous motor, obtain the basic torque and maximum torque coefficient of asynchronous motor and other key parameters, and build the equivalent model of asynchronous motor on the RSCAD draft.

(ii) Obtain the factory test report of synchronous generator, obtain key parameters such as rotor type, pole number, transient, \& sub-transient, \& steady-state reactance, short-circuit characteristic curve, no-load characteristic curve, enter the parameters into the standard synchronous generator model on the RSCAD draft.

(iii) Set up the rotor excitation circuit based on current controlled current source (CCCS) and connect it to the synchronous generator model. Select correspondent exciter system model from IEEE Std 421.5 TM $_{\text {-2005 }}$ according to the actual exciter system.

(iv) Set up the excitation system fault modules, including loss of field module, asynchronous motor fault value and forced excitation module etc. The loss of field module can simulate sudden field loss, proportional field loss, linear\& non-linear field loss etc.

\subsection{Simulation scheme}

(i) Simulate when two M-G sets operating in parallel with different loads, one generator has sudden total filed loss (field current drops to 0 within hundreds of milliseconds).

(ii) Simulate when two M-G sets operating in parallel with different loads, one generator has sudden proportional filed loss (field current drops to $\mathrm{x} \%$ within hundreds of milliseconds).

(iii) Simulate when two M-G sets operating in parallel with different loads, one generator has slow linear\& proportional filed loss (field current drops to 0 within seconds or minutes).

(iv) Simulate when two M-G sets operating in parallel with different loads, one generator has linear\& non-linear force excitation (field current rises to upper limit of the regulator within seconds).

\subsection{Protection\& Control verification}

(i) When one generator has sudden field loss, observe whether the terminal current of another unit running in parallel will exceed the setting value of the (inverse) overcurrent protection, if so, verify the operation sequence of field loss protection.

(ii) When one generator has different proportional field loss, observe whether the terminal current of another unit running in parallel will exceed the setting value of the (inverse)overcurrent protection and at what field loss degree would that happen, then verify the operation sequence of field loss protection and (inverse) overcurrent protection.

(iii) When one generator has different linear\& nonlinear field loss, observe whether the terminal current of another unit running in parallel will exceed the setting value of the (inverse)overcurrent protection and at what field loss speed would that happen, then verify the operation sequence of field loss protection and (inverse) overcurrent protection.

(iv) When two M-G sets operating in parallel with different loads, one generator has forced excitation abnormally. Under the restriction of P-Q limit, observe whether the loss of excitation protection of the other generator acts, and verify the coordination of P-Q limit and loss of excitation protection.

(v) When two M-G sets operating in parallel with different loads, one generator has forced excitation abnormally. Under the restriction of P-Q limit, observe whether the (inverse) overcurrent protection of the other generator acts, and verify the coordination of P-Q limit and (inverse) overcurrent protection. Observe the maximum voltage during the force excitation, verify the validity of the current strategy (trip\& send warnings) against over-voltage.

(vi) During the field loss\& over-excitation, observe the terminal voltage fluctuation to check the PID parameter settings.

\section{Practice}

This chapter apply the modelling method, simulation scheme and protection\& control verification method on an actual M-G sets power supply system.

The major parameters of the generator in the $\mathrm{M}-\mathrm{G}$ set power supply system is shown in the table below:

Table 1. Major parameters of synchronous generator

\begin{tabular}{|c|c|}
\hline Parameter & Value \\
\hline Rated voltage (L-L rms) & $260 \mathrm{~V}$ \\
\hline $\begin{array}{l}\text { Rated apparent power } \\
\text { Number of Poles }\end{array}$ & $\begin{array}{c}\text { 400MVA } \\
4\end{array}$ \\
\hline $\mathrm{D}$ axis reactance $X_{d}$ & 0.83 p.u. \\
\hline $\mathrm{d}$ axis transient reactance ${ }^{X_{d}^{\prime}}$ & 0.36 p.u. \\
\hline $\mathrm{d}$ axis sub-transient reactance $X_{d}^{\prime \prime}$ & 0.15 p.u. \\
\hline Stator leakage reactance $X_{\sigma}$ & 0.04 p.u. \\
\hline q-axis synchronous reactance ${ }^{X_{q}}$ & 0.20 p.u. \\
\hline $\mathrm{q}$-axis sub-transient reactance $X_{q}^{\prime \prime}$ & 0.09 p.u. \\
\hline
\end{tabular}

The synchronous generator applies the self shunt excitation. It adopted the ABB UNITROL 1020 AVR

(Automatic Voltage Regulator), the excitation system diagram is shown in fig 3 .

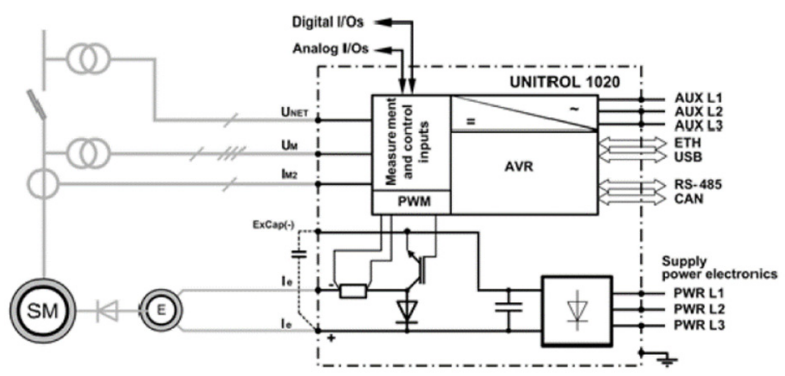

Fig 3. Excitation system with Unitrol 1020 digital AVR 
The parameters of the AVR PID controller is shown in table 2 :

Table 2. PID CONTROLLER PARAMETERS

\begin{tabular}{cccc}
\hline Parameters & Description & Value & Unit \\
\hline VP & Porportional gain & 12 & p.u \\
TA & Integral time constant & 4 & $\mathrm{~s}$ \\
TB & Differential time constant & 0.2 & $\mathrm{~s}$ \\
KB & Controller differential gain & 3 & p.u \\
\hline
\end{tabular}

The load of the M-G sets power supply system is discrete, the load current for two typical different working modes are $100 \mathrm{~A}$ (rated) and 500A (maximum) at the power factor of 0.7 .

To simplify the modelling of asynchronous motor, an ideal torque model is used to provide mechanic torque for the synchronous generator, because the high inertia of motor-flywheel-generator and its totally acceptable to consider the mechanic torque is invariant during the electro-magnetic transient process that last for seconds. Since the mechanic output of the asynchronous motor is relevant to the slip rate, which means the frequency of the generator is not exactly the rated frequency. It is crucial to evaluate the effect that slip rate poses to the accuracy of the simulation results.

The minimum frequency can be calculated as:

$$
\left\{\begin{array}{l}
\omega_{\min }=f\left(P_{\max }\right) \\
f_{\min }=\frac{\omega_{\min }}{2 \pi}
\end{array}\right.
$$

Where the function $\mathrm{f}$ is determined by curve fitting based on the power-revolution speed chart provided by the test report of the asynchronous motor before put into operation. The fitted curve is shown in fig 4.

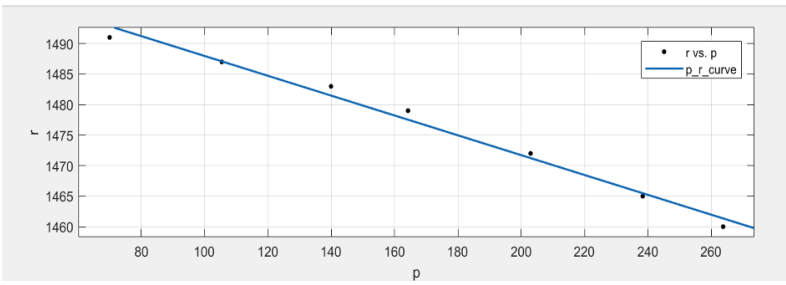

Fig 4. Power-rotational speed fitting curve

It can then be calculated that the minimum frequency is $49.5 \mathrm{~Hz}$ at the maximum load $500 \mathrm{~A}(0.7)$, while the frequency at normal load is $49.8 \mathrm{~Hz}$. During the field loss \& forced excitation simulation, the real power can be considered invariant. So by using ideal torque model\& high inertia governor model and setting frequency before simulation started can simulate the characteristics of the asynchronous motor in the M-G sets simulation.

The synchronous generators have digital protection devices, including over-current protection, field loss protection and over-voltage protection. The AVR has the P-Q controller which is designated to specify the leading phase operation zone. Those protection\& controls should work in coordination to ensure the steady power supply.
The settings of the protection is shown in table 3:

Table 3. Protection settings

\begin{tabular}{cccc}
\hline Protection & Rated Value & $\begin{array}{c}\text { Setting } \\
\text { Value }\end{array}$ & $\begin{array}{c}\text { operation } \\
\text { time }\end{array}$ \\
\hline $\begin{array}{c}\text { Overcurrent } \\
\text { protection }\end{array}$ & $\mathrm{In}=890 \mathrm{~A}$ & $\mathrm{I}>1 \mathrm{In}$ & $0.4 \mathrm{~s}$ \\
$\begin{array}{c}\text { Field loss } \\
\text { protection }\end{array}$ & $\mathrm{Ifdn}=1.6 \mathrm{~A}$ & $\mathrm{I}<0.5 \mathrm{~A}$ & $5 \mathrm{~s}$ \\
$\begin{array}{c}\text { Over voltage } \\
\text { protection }\end{array}$ & $\mathrm{Un}=260 \mathrm{~V}$ & $\mathrm{U}>1.3 \mathrm{Un}$ & $0.4 \mathrm{~s}$ \\
\hline
\end{tabular}

The P-Q limiter operation zone is defined by a list of $\mathrm{P}-\mathrm{Q}$, as shown in table 4 :

Table 4. P-Q limiter settings

\begin{tabular}{|c|c|c|c|c|c|}
\hline & 1 & 2 & 3 & 4 & 5 \\
\hline $\mathrm{P}(\% \mathrm{PN})$ & 0 & 25 & 50 & 75 & 100 \\
\hline $\mathrm{Q}(\% \mathrm{QN})$ & -30 & -25 & -20 & -15 & -10 \\
\hline
\end{tabular}
$5:$

The operation zone defined by the table is shown in fig

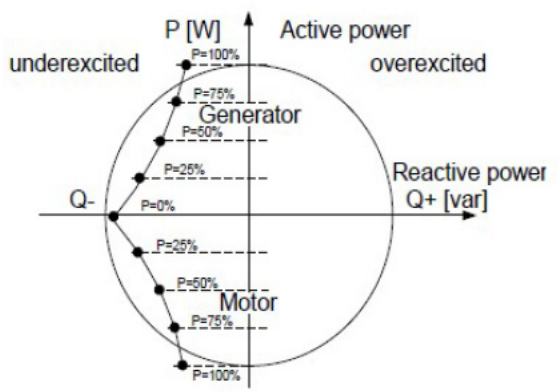

Fig 5. The operation principle of P-Q limiter

After modelling the synchronous machine, asynchronous motor\& excitation system of the M-G sets in RSCAD based on the parameters listed above, all the simulations mentioned in chapter III are conducted.

The simulation results of field loss faults are listed in table 5- table 6:

Table 5. Sudden field loss simulation results

\begin{tabular}{cccccc}
\hline Fault & $\begin{array}{c}\text { Load/Loss } \\
\text { percentage }\end{array}$ & Ig1/A & Ifd1/A & Ig2/A & Ifd2/A \\
\hline $\begin{array}{c}\text { Sudden } \\
\text { field loss of } \\
\text { generator 1 }\end{array}$ & $120 \mathrm{~A} / 100 \%$ & 1138 & 0 & 1138 & 1.42 \\
& $500 \mathrm{~A} / 100 \%$ & 1124 & 0 & 1595 & 1.77 \\
$\begin{array}{c}\text { Sudden } \\
\text { proportional } \\
\text { field loss of } \\
\text { generator 1 }\end{array}$ & $120 \mathrm{~A} / 65.6 \%$ & 380 & 0.5 & 491 & 1.04 \\
\hline
\end{tabular}

Table 6. slow field loss simulation results

\begin{tabular}{ccccc}
\hline Fault & $\begin{array}{c}\text { Load/Field } \\
\text { loss time }\end{array}$ & Ig1/A & Ifd1/A & Ig2/A \\
\hline Liner slow & $0 \mathrm{~A} / 5 \mathrm{~s}$ & 1167 & 0.5 & 1179 \\
field loss of & $120 \mathrm{~A} / 10 \mathrm{~s}$ & 770 & 0.5 & 895 \\
generator1 & $500 \mathrm{~A} / 60 \mathrm{~s}$ & 256 & 0.5 & 883 \\
\hline
\end{tabular}


In table $5 \&$ table $6, \mathrm{~g} 1$, Ifd 1, Ig 2 , Idf2 are the terminal current and field current of generator $1 \& 2$. The terminal current recorded above are the phase current RMS 4 seconds after the field loss fault. It can be concluded that, under different field loss situations, the terminal current of the non-field-loss generator is always greater than the field-loss one, and the maximum fault current is around $1600 \mathrm{~A}$, which is greater than the current setting value of overcurrent protection-888A. Which means the operation of overcurrent protection of the non-field-loss generator would precede the field-loss protection of the field-loss one, causing the two generators trip one after the other. So, its recommended to adopt inverse time overcurrent protection instead, the operation time limit at terminal current $1600 \mathrm{~A}$ should be longer than the time limit of field loss protection $5 \mathrm{~s}$.

The P-Q limiter of the AVR is designated to restrain the leading phase operation level by limiting the field current at specified P-Q work point. When one generator mistakenly increases its field current (Mostly because of PT line break), the other generator would lower its field current to keep terminal voltage at rated level. The P-Q limiter would prevent the field current from further decreasing once the P-Q hit the boundary. It's necessary to find out whether the overcurrent protection\& field loss protection can work in coordination with restrain of $\mathrm{P}-\mathrm{Q}$ limiter when one generator mistakenly increase its field current to the maximum level.

Linearly increase Ifd1 to maximum, records the terminal voltage, the terminal current, maximum ifd 1 and minimum ifd2, as is shown in table 7 :

\begin{tabular}{cccccc}
\multicolumn{5}{c}{ Table 7. mistakenly forced excitation at maximum load } \\
\hline fault & Ifd1.max & Ifd2.min & Ut.max & Ig1.max & Ig2.max \\
\hline $\begin{array}{c}\text { mistakenly } \\
\text { forced } \\
\text { excitation of } \\
\text { generator1 }\end{array}$ & 1.6Ifdn & $1.0 \mathrm{~A}$ & $1.33 \mathrm{Un}$ & $898 \mathrm{~A}$ & $663 \mathrm{~A}$ \\
\hline
\end{tabular}

It can be concluded that when generator 1 mistakenly increases its field current to upper limit, the field current of generator 2 would be greater than the field loss protection setting value $0.5 \mathrm{~A}$. However, the terminal voltage exceeds the setting value 1.3 of the over-voltage protection, which may trigger both generator, which is not acceptable for the requirement of steady and stable power supply. Thus, its recommended that over-voltage protection not trip generators and send warnings instead. The fault should be cut by inverse current protection, and the time limit should be longer than 30s.

To verify the PID controller parameter, records the steady state voltage and voltage callback time after sudden filed loss fault at different load level.

Table 8. PID controller verification

\begin{tabular}{ccc}
\hline $\begin{array}{c}\text { Load } \\
\text { Level }\end{array}$ & $\begin{array}{c}\text { steady state } \\
\text { voltage/\%Un }\end{array}$ & voltage callback time/s \\
\hline $0 \mathrm{a}$ & 94 & 2.5 \\
$120 \mathrm{a}$ & 93 & 6 \\
$500 \mathrm{a}$ & 93 & 7 \\
\hline
\end{tabular}

It can be concluded that the current PID controller parameters can make sure that the voltage could be steady after the field loss fault.

Based on the simulation and analysis above, it can be briefly concluded that the current PID controller and P-Q limiter is reasonable and need no adjustment. However, the over-current protection is advised to be modified to inverse time overcurrent protection, and the time limit should meet the requirement:

Table 9. Inverse time overcurrent settings suggestion

\begin{tabular}{cc}
\hline Terminal current & Time limit \\
\hline $1600 \mathrm{~A}$ & $>5 \mathrm{~s}$ \\
$900 \mathrm{~A}$ & $>20 \sim 30 \mathrm{~s}$ \\
\hline
\end{tabular}

\section{Conclusion}

In this paper, a modelling \&simulation and protection\& control verification method for asynchronous motorsynchronous generator sets (M-G sets) is proposed. The modelling \& simulation method can precisely simulate the electro-magnetic characteristics of the $\mathrm{M}-\mathrm{G}$ sets, making it possible to analyse the fault characteristics under different fault conditions. According to an accident report from a power plant, the improper protection configuration is revealed, and the simulation scheme for the protection\& control verification is then proposed. Lastly, we build the RTDS model for an actual M-G sets in a power plant and conduct simulations of different field loss faults and mistakenly forced excitations, verify the coordination of over-current protection, field loss protection and overvoltage protection, and make proper advice on protection settings. The PID parameters and PQ limiter of the digital AVR are verified as well, which proves that both are reasonable. The method can be applied for all the M-G sets modelling \&simulation and protection\& control verification, ensuring the steady power supply of important loads.

\section{Acknowledgements}

This work is supported by the research and development project of China Electric Power Research Institute: Research on loss of excitation protection technology of generator based on power plane.

\section{References}

1. Jin Jiankun, Li lanwen. Application of pocketcas in coordination of low excitation limit and loss of excitation protection of generator excitation system [J]. Electromechanical information, 2020 (08): 7-8. (in Chinese)

2. Li can, Zhu Hongchao, Yang Ling. Simulation model correction technology of excitation system [J]. Application of motor and control, 2019, 46 (11): 6468. (in Chinese)

3. Ma Wenying, research on protection measures for generator loss of excitation [J]. China Equipment Engineering, 2019 (16): 108-109. (in Chinese) 
4. Xue Lei, Chen Yuanzhi, Kou Shuichao, sun Ganghu, Wu Pengyue, Jiao Shangbin. Steady state asynchronous operation process analysis of $660 \mathrm{MW}$ turbogenerator after loss of excitation [J]. Power system protection and control, 2019, 47 (11): 181-187. (in Chinese)

5. Fan Xiaoming, application research on excitation control system of synchronous generator [J]. Electrical technology, 2018 (20): 70-71. (in Chinese)

6. Wang Jie, sun Zhonghua, Wang Ruicheng. Analysis of excitation system of brushless alternator [J]. Electrical automation, 2018,40 (04): 13-15 + 87. (in Chinese)

7. Li Xiliang. Analysis of common problems of UNITROL 5000 excitation system [J]. Electromechanical information, 2018 (18): 8-9. (in Chinese)

8. Liu Nian, Xie Chi, Zhao Xin, pan Rongchao, Huang Dake. Analysis and Research on forced excitation characteristics of synchronous generator superimposed forced excitation system [J]. Sichuan electric power technology, 2017,40 (06): 20-23. (in Chinese) 